\section{MS6-P5 Helical Foldamers as Hosts for Rod-like Guests}

Barbara Wicher ${ }^{1}$, Xiang Wang ${ }^{1}$, Yann Ferrand ${ }^{1}$, Ivan Huc ${ }^{1}$

1. Univ. Bordeaux, CBMN, UMR 5248, Institut Européen de Chimie Biologie, 2 rue Escarpit 33607 Pessac, France and CNRS, CBMN, UMR 5248, Institut Européen de Chimie Biologie, 2 rue Escarpit 33607 Pessac,

email: b.wicher@iecb.u-bordeaux.fr

Biomolecular machines, such as kinesin or the ribosome, have always been a paragon for chemists trying to obtain more and more advanced supramolecular architectures. During decades of research, organic chemists have developed a wide range of prototypes of artificial molecular machines. However, the design and synthesis of molecules able to execute particular tasks remains a challenge. ${ }^{[1]}$

During the course of our studies we have synthesized aromatic oligoamide foldamers able to fold and self-assemble into double helices. Their inner cylindrical hollow was designed to be complementary to linear oligourethanes around which the helices can wind. ${ }^{[2,3]}$ Kinetic studies showed that these foldamers can shuttle along rod-like guest molecules in a sort of sliding motion with varying rates according to the length of the rods. We showed that the molecular structure of the guest determines the supramolecular pathway used by the helical shuttle.

In this poster, we will present several crystal structures of an oligoamide double helix forming pseudo-rotaxane architectures upon winding around rod-like guest molecules. X-ray studies revealed that helix-rod intermolecular hydrogen bonds and also the shape and size of the internal cavity of the double helix can adapt to accommodate differences in the structure of guest molecules. This flexibility allows the same oligoamide helix to be an excellent host for a large variety of guest molecules.

[1] Erbas-Cakmak et al (2015), Chem. Rev. 115, 10081-10206.

[2] Gan et al (2011), Science, 331, 1172-1175.

[3] Ferrand et al (2011), Agew. Chem. Int. Ed. 50, 7572-7575.

This work was supported by European Research Council under the European Union's Seventh Framework Programme (grant agreement no. ERC-2012-AdG-320892, post-doctoral fellowship to B.W.)
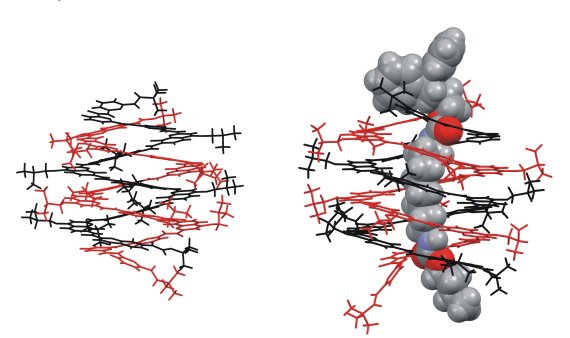

Figure 1. Oligoamide in a double helix form without (left) and with (right) rod-like guest molecule.
Keywords: helical foldamers, oligourethanes, X-ray studies 